\title{
ARTÍCULO ORIGINAL \\ Perfiles de salud en pacientes con enfermedades crónicas en una institución de salud de alta complejidad
}

\section{Health profiles in patients with chronic diseases in a high- complexity health institution / Perfis de saúde em pacientes com doenças crônicas numa instituição de saúde de alta complexidade}

Juan Guillermo Barrientos Gómez ${ }^{1,2}$, Laura María Pineda Jiménez², Natalia Zapata Zuluaga², Aura María Vélez Granda², María Manuela Echavarría Noreña², Isabella Montoya Botero $^{2}$

\section{RESUMEN}

Objetivo: describir los diferentes perfiles, en términos de calidad de vida relacionada con la salud, en pacientes con enfermedades crónicas en una institución de salud de alta complejidad.

Metodología: estudio transversal descriptivo. Se evaluó la calidad de vida relacionada con la salud mediante el instrumento EQ-5D en pacientes con enfermedades crónicas que consultaron a medicina interna y sus subespecialidades, en el servicio de consulta externa de una institución de salud de alta complejidad de la ciudad de Medellín durante el año 2017.

Resultados: fueron encuestados 158 pacientes. La mediana de la edad fue 58 (46-68) años. El tiempo aproximado de evolución entre la edad de realización del diagnóstico principal y la edad del paciente al momento de la encuesta fue de 8.7 años, un total de 117 pacientes (74.1\%) eran de sexo femenino. El estado de salud general de los pacientes de endocrinología y reumatología fue de 80 puntos, siendo los factores que afectaron la calidad de vida los denominados "dolor o malestar" y "ansiedad o depresión". Además, en los pacientes con patologías infecciosas el estado de salud general fue el más bajo, 62.5 , donde el factor "dolor o malestar" fue el más común.

Conclusiones: se identifica mayor alteración en las variables del EQ-5D de "dolor o malestar" y "ansiedad o depresión" en la población general, así mismo, cuando se trata del sexo femenino y de las áreas de endocrinología y reumatología.

Palabras clave: calidad de vida relacionada con la salud; perfil de salud; enfermedad crónica; endocrinología; reumatología

\section{ABSTRACT}

Objective: to describe the different profiles in terms of health-related quality of life found in patients with chronic diseases in a high-complexity health institution.

Methodology: cross-sectional descriptive study that evaluated the health-related quality of life through the EQ-5D instrument, in patients with chronic diseases that consulted Internal Medicine and its subspecialties in the outpatient service of a highly-complexity health institution in the city of Medellin, during the year 2017.

Results: 158 patients between the ages 46-68 (an average of 58-year-old) were surveyed. A total of 117 patients (74.1\%) were female. The time of evolution from the age when the diagnostic was run to the actual age of the patient when surveyed was 8.7 years.

The general health status of the patients from Endocrinology and Rheumatology was found to be of 80 points, being the feelings of "pain or discomfort" and "anxiety or depression" the ones that most affected the quality of life.
Fecha de recibido: 18 de marzo de 2019

Fecha de aprobación: 10 de septiembre de 2019

Forma de citar este artículo: Barrientos JG, Pineda LM, Zapata N, Vélez AM, Echavarría MM, Montoya I. Perfiles de salud en pacientes con enfermedades crónicas en una institución de salud de alta complejidad. Med UPB. 2020;39(1):13-22.

DOI:10.18566/medupb.v39n1.a04

$1 \quad$ Clínica Universitaria Bolivariana. Medellín, Colombia.

2 Facultad de Medicina Universidad Pontificia Bolivariana. Medellín, Colombia.

Dirección de correspondencia: Juan Guillermo Barrientos Gómez. Correo electrónico: juan.barrientos@upb.edu.co 
Additionally, in patients with infectious pathologies the general health status was the lowest (62.5); the most common factor was "pain or discomfort).

Conclusions: it is evident that there is greater alteration in the variables of the EQ-5D of "pain or discomfort" and "anxiety or depression" in the general population, as well as in the females and the areas of Endocrinology and Rheumatology.

Keywords: health related quality of life; health profile; chronic disease; endocrinology; rheumatology

\section{RESUMO}

Objetivo: descrever os diferentes perfis, em termos de qualidade de vida relacionada com a saúde, em pacientes com doenças crônicas numa instituição de saúde de alta complexidade.

Metodologia: estudo transversal descritivo. Se avaliou a qualidade de vida relacionada com a saúde mediante o instrumento EQ-5D em pacientes com doenças crônicas que consultaram a medicina interna e suas subespecialidades, no serviço de consulta externa de uma instituição de saúde de alta complexidade da cidade de Medellín durante o ano 2017.

Resultados: foram entrevistados 158 pacientes. A média de idade foi 58 (46-68) anos. O tempo aproximado de evolução entre a idade de realização do diagnóstico principal e a idade do paciente ao momento da enquete foi de 8.7 anos, um total de 117 pacientes (74.1\%) eram de sexo feminino. $O$ estado de saúde geral dos pacientes de endocrinologia e reumatologia foi de 80 pontos, sendo os fatores que afetaram a qualidade de vida os denominados "dores ou mal-estar" e "ansiedade ou depressão". Ademais, nos pacientes com patologias infecciosas o estado de saúde geral foi o mais baixo, 62.5 , donde o fator "dor ou mal-estar" foi o mais comum.

Conclusões: se identifica maior alteração nas variáveis de EQ-5D de "dor ou mal-estar" e "ansiedade ou depressão" na população geral, assim mesmo, quando se trata do sexo feminino e das áreas de endocrinologia e reumatologia.

Palavras chave: qualidade de vida relacionada com a saúde; perfil de saúde; doença crônica; endocrinologia; reumatologia

\section{INTRODUCCIÓN}

La evaluación de los resultados en salud es un proceso que tiene como objetivo generar evidencias que permitan a los gestores tomar decisiones con base en un conocimiento previo de una situación, con el propósito de crear estrategias de mejoramiento de las limitaciones que se identifiquen. Brinda también información sobre los fenómenos relacionados con los servicios de salud y permite medir la eficacia y calidad en la atención, para la toma de decisiones sobre actividades del sector $^{1}$. Estas estrategias se han logrado implementar gracias a la investigación en servicios de salud, definida como la investigación científica encargada de medir y analizar los resultados en salud, con el objetivo principal de ofrecer herramientas para una toma de decisiones acertadas que promuevan el bienestar de los pacientes y que logren la excelencia en la calidad asistencial ${ }^{2,3}$.

La Organización Mundial de la Salud (OMS) define la calidad de vida (CV) como "el estado de completo bienestar físico, mental y social, y no solamente la ausencia de enfermedad"4. Por otro lado, la calidad de vida relacionada con la salud (CVRS) es multidimensional e incluye aspectos subjetivos y objetivos, que involucran el efecto de la enfermedad sobre el contexto personal, familiar y social. E1 paciente da su propia percepción de su salud, teniendo en cuenta: el funcionamiento físico, social, cognitivo, emocional y el cuidado personal ${ }^{5}$. Para esta valoración se han desarrollado múltiples instrumentos clasificados como específicos (centrados 
en una enfermedad o síndrome concreto) y generales (aplicados a cualquier tipo de población, independiente del diagnóstico $)^{6-16}$.

A pesar de lo anterior, en la actualidad la medición de resultados en salud se calcula mediante parámetros clínicos, donde el paciente es evaluado solo en su dimensión física, sin ocuparse de las dimensiones sociales, cognitivas y emocionales; esto conduce a la toma de decisiones sin tener en cuenta la CVRS, omitiendo aspectos fundamentales del paciente como ser integral ${ }^{8}$. De ahí nace la necesidad de conocer en primera instancia en qué estado de salud se encuentran los pacientes, lo que significa conocer cuál es la CVRS antes de realizar algún procedimiento médico, para posteriormente observar cómo mejoró la situación, y de esta manera poder implementar medidas que impacten sobre dimensiones del paciente que influyen sobre el tratamiento,la adherencia, la adaptación y el seguimiento de la enfermedad ${ }^{3,13,17}$.

La investigación en servicios de salud, enfocada sobre los resultados centrados en el paciente (humanísticos), abre un nuevo paradigma que deja de lado la evaluación de los resultados clínicos (enfoque tradicional biológicofuncional). El enfoque humanista implica pensar que los resultados percibidos por los pacientes son indicadores de impacto en su CVRS ${ }^{10,14,18}$. En Colombia se han realizado estudios sobre los perfiles de CVRS en los pacientes con patologías crónicas ${ }^{19-35}$, mediante instrumentos específicos para cada enfermedad; pero pocos estudios se han realizado con instrumentos genéricos que permitan comparar las enfermedades crónicas y el estado actual de cada una, respecto a las otras. Por esta razón se realiza este estudio para describir los diferentes perfiles en términos de CVRS, en pacientes con patologías crónicas en una institución de salud de alta complejidad, con el fin de poder generar insumos para tomar decisiones, no solo basadas en parámetros clínicos, sino también teniendo en cuenta la CVRS.

\section{METODOLOGÍA}

\section{Tipo de estudio, población y muestra}

Estudio transversal descriptivo, en el cual se incluyeron pacientes mayores de edad con patologías crónicas que consultaron a medicina interna y sus subespecialidades (endocrinología, reumatología, neurología, infectología y neumología) en el servicio de consulta externa de una institución de salud de alta complejidad de la ciudad de Medellín, durante el año 2017. Se excluyeron aquellos con problemas cognitivos o psiquiátricos que impidieran la aplicación del instrumento y los que no dieron su consentimiento. El cálculo de la muestra se realizó con base en el número de atenciones realizadas en el servicio durante el año previo (2016) según la subespecialidad (endocrinología 1855 , reumatología 1301 , neurología 837 , infectología 408 , neumología 260 y medicina interna 70). Fueron en total 4731 atenciones; con una confianza del $95 \%$, un error fijado en el 10\% y una prevalencia del riesgo del 80\%; por tanto, la muestra distribuida según la subespecialidad fue: endocrinología 62, reumatología 43, neurología 28 , infectología 14 , neumología 9 y medicina interna 2, para un total de 158 encuestados. La selección fue no aleatoria, según la disponibilidad de pacientes en el momento de la encuesta.

\section{Instrumentos de recolección y variables}

Después de realizar la estandarización del protocolo de investigación, la información fue recolectada por los investigadores (LMPJ, NZZ, AMV, MMEN e IMB), mediante encuestas diligenciadas por los pacientes. En la primera parte de la encuesta se indagaba acerca de variables como: edad, sexo, estrato socioeconómico, años de realización del diagnóstico principal, diagnóstico principal, comorbilidades y tipo de tratamiento recibido a la fecha. Posteriormente, se aplicó el instrumento EQ5D para evaluar la percepción de la salud frente a una enfermedad, a través de cinco dimensiones y teniendo en cuenta aspectos de la vida diaria (movilidad, cuidado personal, actividades cotidianas, dolor/malestar y ansiedad/depresión). El EQ-5D cataloga los pacientes en 243 estados de salud que describen las características de la $\mathrm{CVRS}^{36}$. El Instituto de Evaluación de Tecnologías en Salud (IETS) de Colombia recomienda este instrumento genérico para la evaluación de los efectos o resultados en evaluaciones económicas y en el estudio de la CVRS. El EQ-5D es corto y sencillo de diligenciar (en 2 o 3 minutos), implica poca carga cognitiva para el encuestado, puede utilizarse tanto en individuos relativamente sanos (población general) como en grupos con diferentes patologías. Está validado en población latina ${ }^{10,37,38}$. Como desventaja, al ser un instrumento genérico, su capacidad discriminativa y su sensibilidad, con relación a un instrumento específico, es inferior ${ }^{10,37,38}$.

La clasificación internacional de enfermedades (CIE10) se usó para registrar los diagnósticos principales y el número de comorbilidades de los pacientes. Esta clasificación es usada en el mundo para recolectar información en los diferentes niveles de atención en salud con fines epidemiológicos, de gestión sanitaria y uso clínico ${ }^{39}$.

\section{Procesamiento de la información y análisis de datos}

A partir de los datos recolectados se creó una base de datos en Excel 2016 (Microsoft-Office ${ }^{\circledR}$ ). Las variables cualitativas se reportan en frecuencias absolutas y rela- 
tivas. Para las variables cuantitativas se usan medidas de tendencia central (media o mediana) y de dispersión (desviación estándar o rango intercuartílico) según su distribución (prueba de normalidad de KolomogorovSmirnov). Se empleó SPSS 21.0.

\section{Consideraciones éticas}

La investigación se realizó siguiendo los lineamientos de la Declaración de Helsinki de 2013 y la Resolución 008430 de 1993 del Ministerio de Salud de la República de Colombia. Este proyecto fue considerado de riesgo mínimo, ya que ciertos ítems del instrumento EQ-5D abarcan temas respecto a la situación emocional y física. Se obtuvo la aprobación del Comité de Ética de Investigación en Salud de la Universidad Pontificia Bolivariana (acta N.o 2 del año 2017). Los participantes dieron su consentimiento por escrito y se veló por la confidencialidad de los datos.

\section{RESULTADOS}

Fueron evaluados 158 pacientes con patologías crónicas. La mediana de la edad fue 58 (46 - 68) años y el promedio de edad de realización del diagnóstico principal fue $49.3 \pm 18.7$ años. Un total de 117 pacientes (74.1\%) eran de sexo femenino. La distribución del estrato socioeconómico fue de 18 (11.4\%), 44 (27.8\%), 47 (29.7\%), $24(15.2 \%), 20(12.7 \%)$ y $5(3.2 \%)$ para los estratos del uno al seis, respectivamente.

En la Tabla 1 se describen los diagnósticos principales y el número de comorbilidades de los pacientes según la CIE-10.

En la Tabla 2 se encuentran los resultados de cada una de las variables que evalúa la encuesta EQ-5D.

Se presentan en la Tabla 3 y en la Tabla 4 los resultados de cada una de las variables que evalúa la EQ-5D, según sexo y subespecialidad: endocrinología, reumatología, neurología, infectología, neumología y medicina interna, respectivamente.

De los 158 pacientes, 106 (67\%) practicaban hábitos de vida saludable, 144 (91.1\%) recibían tratamiento médico y 35 (22.1\%) han requerido tratamiento quirúrgico para el manejo del diagnóstico principal por el cual consultaban a la subespecialidad.

\section{DISCUSIÓN}

La aplicación de instrumentos que evalúen la CVRS en las enfermedades, permite percibir la panorámica del

Tabla 1. Distribución de los diagnósticos principales y morbilidades según la clasificación CIE-10.

\begin{tabular}{lc}
\hline \multicolumn{1}{c}{ Diagnóstico principal } \\
\hline \multicolumn{2}{c}{ Variable (n = 158) } \\
\hline Enfermedad endocrina, $\mathbf{n}(\%)$ & Estadístico \\
$\quad$ Trastornos tiroideos: E039, E042, E890, E059, R946, E069 & $15(9.4 \%)$ \\
Diabetes mellitus: E109, E118, E119, E138, E139, E147 & $13(8.2 \%)$ \\
Osteoporosis: M810, M805, M809 & $13(8.2 \%)$ \\
Tumor maligno de la glándula tiroides: C73X & $10(6.3 \%)$ \\
Obesidad: E669, E660 & $5(3.1 \%)$ \\
Otros: E220, F529, E893, E782, E260, E271, E210, E213, E221, E559 & $14(8.8 \%)$ \\
Total, n (\%) & $70(44.3 \%)$ \\
\hline Enfermedad autoinmune, n (\%) & \\
Artropatía por cristales: M119 & $5(3.1 \%)$ \\
Osteoartrosis primaria generalizada: M150 & $5(3.1 \%)$ \\
Artritis reumatoide: M058, M059, M060 & $4(2.5 \%)$ \\
Espondilopatía inflamatoria: M469 & $3(1.8 \%)$ \\
Síndrome seco [sjögren]: M350 & $3(1.8 \%)$ \\
Otros: I730, M313, M790, M791, M075, M331, M321, M353, M359 & $11(6.9 \%)$ \\
Total, n (\%) & $31(19.6 \%)$ \\
\hline Enfermedad neurológica y mental, n (\%) & $15(9.4 \%)$ \\
Cefalea y migraña: R51, G442, G443, G430, G431, G433, G448 & $3(1.8 \%)$ \\
Epilepsia: G401, G409 & $7(4.4 \%)$ \\
Otros: F03, G454, R268, G629, M961, R42, F412 & $25(15.8 \%)$ \\
\hline
\end{tabular}


Enfermedad infecciosa, $\mathbf{n}(\%)$

Infección de vías urinarias: N390 $\quad 4(2.5 \%)$

Tuberculosis: A151, Z201 $2(1.2 \%)$

Infección del sistema nervioso central: G009, G049 $2(1.2 \%)$

Otros: B399, A799, Z226, M886, B333 $5(3.1 \%)$

Total, $\mathrm{n}(\%)$

$13(8.2 \%)$

Enfermedad del sistema respiratorio, n (\%)

Enfermedad pulmonar obstructiva crónica y asma: J42, J449, J459, J451 5 (3.1\%)

Apnea del sueño: G473 $\quad 2(1.2 \%)$

Otros: I270, R05, J849 $3(1.8 \%)$

Total, n (\%)

10 (6.3\%)

Enfermedad del aparato locomotor, lesiones y traumatismos, $\mathrm{n}$ (\%)

Traumatismo intracraneal y secuelas: S069, T905 $4(2.5 \%)$

Artrosis: M199

$3(1.8 \%)$

Otros: S220, M255 $2(1.2 \%)$

Total, n (\%)

$9(5.6 \%)$

\begin{tabular}{ccc}
\hline \multicolumn{1}{c}{ Variable $(\mathbf{n}=\mathbf{1 5 8})$} & Morbilidades & Total de morbilidades \\
\hline Rangos de morbilidades, $\mathbf{n}(\%)$ & Pacientes & $109(14.9 \%)$ \\
1 a 3 & $55(34.8 \%)$ & $347(47.5 \%)$ \\
4 a 6 & $70(44.3 \%)$ & $209(28.6 \%)$ \\
7 a 9 & $27(17.0 \%)$ & $64(8.7 \%)$ \\
10 a 12 & $6(3.7 \%)$ & $729(100 \%)$ \\
Total, $\mathbf{n}(\%)$ & $158(100 \%)$ & $(5 \%)$ \\
\hline
\end{tabular}

Tabla 2. Resultado de cada variable de la encuesta EQ-5D para la población en general.

\begin{tabular}{|c|c|}
\hline Variable $(n=158)$ & Estadístico \\
\hline \multicolumn{2}{|l|}{ Movilidad, n (\%) } \\
\hline No tengo problemas para caminar & $115(72.8 \%)$ \\
\hline Tengo algunos problemas para caminar & $39(24.7 \%)$ \\
\hline Tengo que estar en la cama & $4(2.5 \%)$ \\
\hline \multicolumn{2}{|l|}{ Cuidado personal, n (\%) } \\
\hline No tengo problemas con el cuidado personal & $139(88 \%)$ \\
\hline Tengo algunos problemas para lavarme o vestirme solo & $14(8.9 \%)$ \\
\hline Soy incapaz de lavarme o vestirme solo & $5(3.2 \%)$ \\
\hline \multicolumn{2}{|l|}{ Actividades de todos los días, n (\%) } \\
\hline No tengo problemas para realizar mis ATD* & $106(67.1 \%)$ \\
\hline Tengo algunos problemas para realizar mis ATD & $40(25.3 \%)$ \\
\hline Soy incapaz de realizar mis ATD & $12(7.6 \%)$ \\
\hline \multicolumn{2}{|l|}{ Dolor o malestar, n (\%) } \\
\hline No tengo dolor ni malestar & $50(31.6 \%)$ \\
\hline Tengo dolor o malestar moderado & 55 (34.8\%) \\
\hline Tengo mucho dolor o malestar & $53(33.5 \%)$ \\
\hline \multicolumn{2}{|l|}{ Ansiedad o depresión, n (\%) } \\
\hline No estoy ansioso ni deprimido & 66 (41.8\%) \\
\hline Estoy moderadamente ansioso o deprimido & 53 (33.5\%) \\
\hline Estoy muy ansioso o deprimido & $39(24.7 \%)$ \\
\hline Variable $(\mathbf{n}=158)$ & Estadístico \\
\hline Estado de salud, promedio $\pm \mathrm{DE}$ & $75.1 \pm 21.2$ \\
\hline
\end{tabular}

*ATD: Actividades de todos los días 
Tabla 3. Resultado según el sexo para cada variable de la encuesta EQ-5D

\begin{tabular}{|c|c|c|c|}
\hline \multirow[b]{2}{*}{ Variable } & \multicolumn{2}{|c|}{ Sexo } & \multirow[b]{2}{*}{$\begin{array}{c}\text { Total } \\
(\mathrm{n}=158)\end{array}$} \\
\hline & $\begin{array}{c}\text { Femenino } \\
(n=117)\end{array}$ & $\begin{array}{c}\text { Masculino } \\
(\mathrm{n}=41)\end{array}$ & \\
\hline \multicolumn{4}{|l|}{ Movilidad, n (\%) } \\
\hline No tengo problemas para caminar & $86(54.4 \%)$ & $29(18.4 \%)$ & $115(72.8 \%)$ \\
\hline Tengo algunos problemas para caminar & $27(17.1 \%)$ & $12(7.6 \%)$ & 39 (24.7\%) \\
\hline Tengo que estar en la cama & $4(2.5 \%)$ & $0(0 \%)$ & $4(2.5 \%)$ \\
\hline Total, n (\%) & 117 (74.1\%) & $41(25.9 \%)$ & $158(100 \%)$ \\
\hline \multicolumn{4}{|l|}{ Cuidado personal, n (\%) } \\
\hline No tengo problemas con el cuidado personal & 104 (65.8\%) & 35 (22.2\%) & 139 (88.0\%) \\
\hline Tengo algunos problemas para lavarme o vestirme solo & $10(6.3 \%)$ & $4(2.5 \%)$ & $14(8.9 \%)$ \\
\hline Soy incapaz de lavarme o vestirme solo & $3(1.9 \%)$ & $2(1.3 \%)$ & $5(3.2 \%)$ \\
\hline Total, $\mathrm{n}(\%)$ & $117(74.1 \%)$ & $41(25.9 \%)$ & $158(100 \%)$ \\
\hline \multicolumn{4}{|l|}{ Actividades de todos los días, n (\%) } \\
\hline No tengo problemas para realizar mis ATD* & 77 (48.7\%) & 29 (18.4\%) & 106 (67.1\%) \\
\hline Tengo algunos problemas para realizar mis ATD & $32(20.3 \%)$ & $8(5.1 \%)$ & 40 (25.3\%) \\
\hline Soy incapaz de realizar mis ATD & $8(5.1 \%)$ & $4(2.5 \%)$ & $12(7.6 \%)$ \\
\hline Total, n (\%) & $117(74.1 \%)$ & $41(25.9 \%)$ & $158(100 \%)$ \\
\hline \multicolumn{4}{|l|}{ Dolor o malestar, $\mathrm{n}(\%)$} \\
\hline No tengo dolor ni malestar & 35 (22.2\%) & $15(9.5 \%)$ & $50(31.6 \%)$ \\
\hline Tengo dolor o malestar moderado & 39 (24.7\%) & $16(10.1 \%)$ & 55 (34.8\%) \\
\hline Tengo mucho dolor o malestar & $43(27.2 \%)$ & $10(6.3 \%)$ & $53(33.5 \%)$ \\
\hline Total, n (\%) & 117 (74.1\%) & $41(25.9 \%)$ & $158(100 \%)$ \\
\hline \multicolumn{4}{|l|}{ Ansiedad o depresión, n (\%) } \\
\hline No estoy ansioso ni deprimido & 47 (29.7\%) & 19 (12.0\%) & 66 (41.8\%) \\
\hline Estoy moderadamente ansioso o deprimido & 39 (24.7\%) & $14(8.9 \%)$ & 53 (33.5\%) \\
\hline Estoy muy ansioso o deprimido & 31 (19.6\%) & $8(5.1 \%)$ & 39 (24.7\%) \\
\hline \multirow[t]{2}{*}{ Total, n (\%) } & 117 (74.1\%) & $41(25.9 \%)$ & $158(100 \%)$ \\
\hline & \multicolumn{3}{|c|}{ Sexo } \\
\hline Variable $(n=158)$ & $\begin{array}{c}\text { Femenino } \\
(n=117)\end{array}$ & & $\begin{array}{l}\text { ulino } \\
41)\end{array}$ \\
\hline Estado de salud, Mediana y RIC & $80(62.5-90)$ & & $-90)$ \\
\hline
\end{tabular}

* ATD: Actividades de todos los días

paciente en diversos momentos de la vida y de la historia natural de su problema médico. Esto cobra importancia al permitir conocer los perfiles de salud y otorgar una visión del proceso de adaptación en los casos crónicos. Tratar al paciente en cada una de las etapas de su enfermedad, teniendo en cuenta no solo su estado biológico sino también su $\mathrm{CV}$, abre a los clínicos oportunidades de tratamiento para el mejoramiento oportuno y satisfactorio de la salud ${ }^{13}$.

En los encuestados, el tiempo aproximado de evolución entre la realización del diagnóstico y la edad del paciente al momento de la encuesta fue de 8.7 años, lo cual coincide con la definición de "enfermedad crónica" de la OMS (afecciones de más de 6 meses y de progresión generalmente lenta $)^{40}$. No obstante, existen otras entidades sanitarias que hablan de cronicidad, incluso desde los tres meses ${ }^{41}$. En este trabajo, hubo predominio de los estratos socioeconómicos dos, tres y cuatro (en casi dos tercios de los encuestados), al igual que del sexo femenino.

En el presente estudio, la distribución de los diagnósticos principales se dio principalmente entre las enfermedades endocrinas y autoinmunes. Probablemente porque en el muestreo se incluyeron más pacientes con patologías crónicas de las subespecialidades de endocrinología y reumatología, siendo una limitación del reporte. Sin embargo, nuestras distribuciones de frecuencia coinciden con las estadísticas mundiales, puesto que, según la OMS, entre las enfermedades crónicas que representan una porción sustancial de la carga mundial de morbilidad está la diabetes, entre otras como las cardiocerebrovasculares o el cáncer ${ }^{42}$. Así mismo, en América Latina y el Caribe entre 1990 y 2010 se ha presentado 


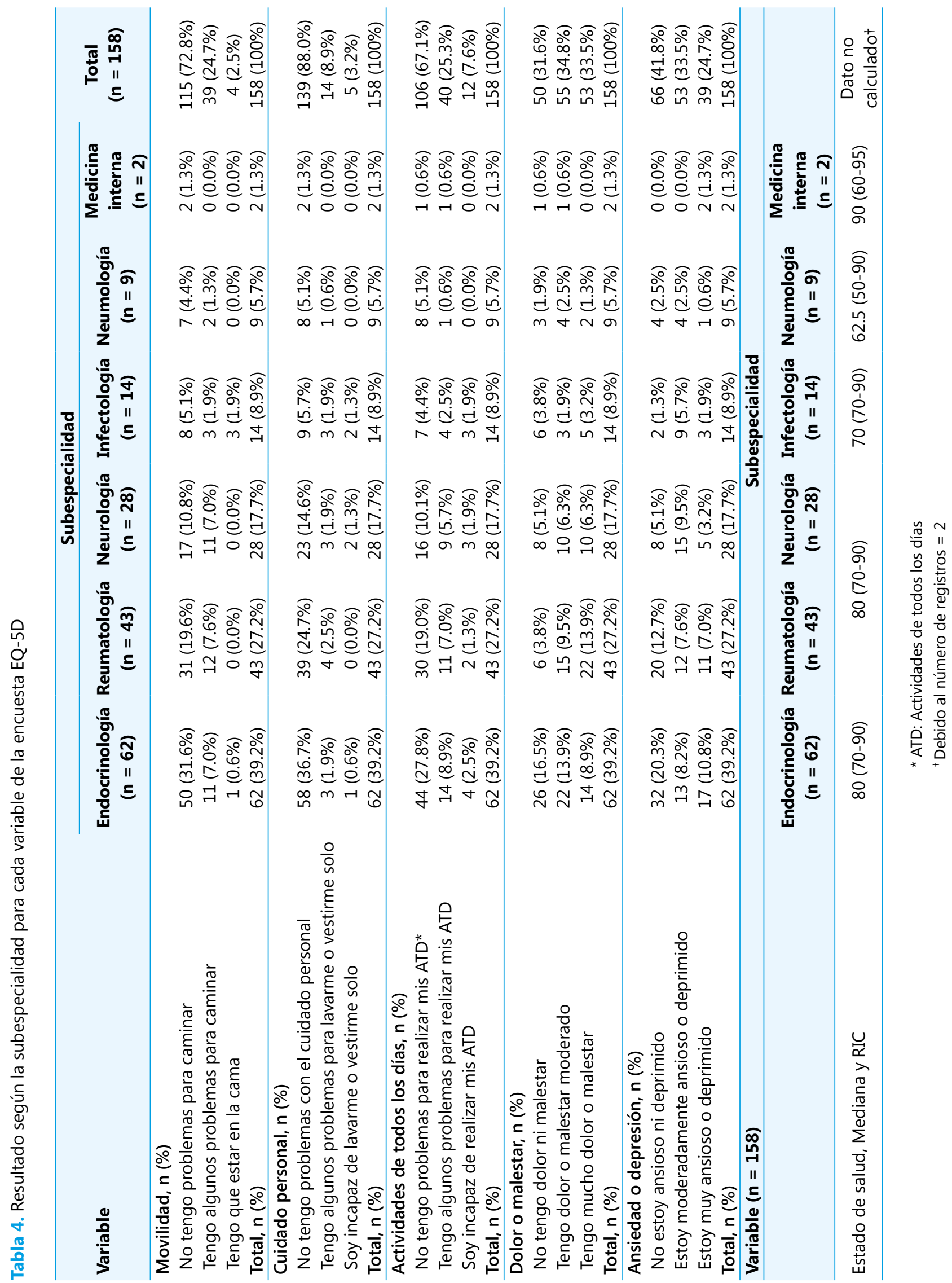


un aumento de la carga de morbilidad causado por las enfermedades osteomusculares y reumatológicas, entre otras no transmisibles, como la cardiopatía isquémica o los trastornos mentales (depresión y ansiedad) ${ }^{43}$.

La multimorbilidad es la presencia de dos o más condiciones de salud crónicas simultáneas en un individuo o población ${ }^{44,45}$.En el presente estudio, cerca del $45 \%$ de los pacientes presentaban entre cuatro y seis condiciones de salud de forma simultánea. Lo anterior en concordancia con la literatura, puesto que en otros estudios se estima que el promedio de problemas médicos por paciente es de cinco. A su vez, el promedio es mayor en pacientes de medicina interna, 5.8, y en mayores de 65 años, 6.4 $4^{46-48}$.

Cuando se analizan las variables más afectadas en el EQ-5D en la muestra y según el sexo y la subespecialidad, se evidencia mayor alteración en las variables de "dolor o malestar" " “ansiedad o depresión" en la muestra general, en las mujeres y en las áreas de endocrinología y reumatología. Esto es contrario a lo identificado en un estudio en pacientes con lupus eritematoso sistémico (LES), que determinó la CVRS a través de la aplicación del instrumento SF-36 y que encontró poca ansiedad y depresión y reducida interferencia del dolor corporal en las actividades de trabajo y de hogar ${ }^{33}$. Otro estudio que también evaluó la CV en pacientes con LES mediante el SF-36, reporta resultados particularmente bajos en el rol físico y el rol emocional, mientras que obtuvo resultados más altos en el funcionamiento físico, funcionamiento social y salud mental ${ }^{49}$.

A continuación se decriben dos estudios que coinciden con lo encontrado en la presente investigación: el primero determinó la $\mathrm{CV}$ en pacientes colombianos con artritis reumatoide, mediante la versión española del cuestionario de Calidad de Vida en la Artritis (QOL-RA). Los puntajes más bajos corresponden a los ítems de dolor y tensión nerviosa ${ }^{23}$. El segundo evaluó la $\mathrm{CV}$ a través del SF-36 en pacientes con diabetes mellitus tipo II, y encontró que la variable sexo ejerce influencia sobre los resultados obtenidos en función física, dolor corporal, salud general, vitalidad y salud mental ${ }^{32}$.

En cuanto a las limitaciones del estudio, se debe aclarar que los resultados pudieron ser influenciados por la forma en la que se llevó a cabo el cálculo de la muestra. Adicionalmente, respecto al sexo, pudo incidir el hecho de que las mujeres son más proclives a responder la encuesta y a consultar con mayor frecuencia. Es así, como los resultados del presente estudio no deben generalizarse y quedan circuncritos a esta muestra.

En conclusión, se identifica mayor alteración en las variables del EQ-5D "dolor o malestar" y "ansiedad o depresión" en la muestra, en el sexo femenino y en las áreas de endocrinología y reumatología. Estos resultados servirán de insumo para futuras investigaciones que validen conductas en la atención y formas de tratamiento en pacientes con enfermedades crónicas, basados en perfiles de salud.

\section{DECLARACIÓN DE CONFLICTO DE INTERESES}

Los autores declaran no tener ningún conflicto de intereses.

\section{REFERENCIAS}

1. Saldivia S, Vicente B, Torres F. Medición de resultados en evaluaciones económicas. Rev Médica Chile. 2010; 138:79-82.

2. Lázaro P, Pozo F, Ricoy JR. Una estrategia de investigación en el sistema nacional de salud: II. Investigación en servicios de salud. Med Clin Barc. 1995; 104(2):67-76.

3. Soto J. Implicación de la investigación de resultados en salud en la mejora continua de la calidad asistencial del Sistema Nacional de Salud. An Med Interna. 2007; 24(11):517-9.

4. Grupo de la OMS sobre la calidad de vida. Qué calidad de vida. Foro Mundial de la Salud. Rev Inter Desar Sanit. 1996; 17:385-7.

5. Gómez M, Sabeh E. Calidad de vida. Evolución del concepto y su influencia en la investigación y la práctica. Universidad de Salamanca [Internet]. 2001 [citado 9 de octubre de 2018]. Disponible en: http://campus.usal.es/ inico/investigacion/ invesinico/calidad.htm.

6. Badia X, Lizán L. Estudios de calidad de vida. En: Martín A, Cano JF (eds), Atención Primaria: conceptos, organización y práctica clínica. Madrid: Elsevier 2003; (I):250-61.

7. Weinstein MC, Torrance G, McGuire A. QALYs: the basics. Value Health. 2009; 12 Suppl 1:S5-9.

8. Llach XB, Tudela LL. Reflexiones sobre la investigación de resultados en salud. Aten Primaria. 2002; 30(6):388-91.

9. Seuc AH, Domínguez E. Introducción al cálculo de esperanza de vida ajustada por discapacidad. Rev Cuba Hig Epidemiol. 2002; 40(2):95-102. 
10. Cañon Ó, Rodríguez C. Introducción al uso de QALYs y EQ-5D en la evaluación de tecnologías en Colombia. CIFE. 2011; 13(19):33-49.

11. Chicaíza-Becerra L, García-Molina M, Gómez GR. Análisis costo utilidad: evolución, fundamentos e implicaciones. Coyuntura Económica. 2013; 43(2):97-111.

12. García F, Alfaro A, Moreno JL. Evaluación de resultados de salud: panorama sobre el uso de medidas de resultados de salud basadas en el paciente en la práctica clínica. Rev Clínica Med Fam. 2009; 2(6):286-93.

13. Yepes-Núñez JJ, García G, Iván H. Preferences of states of health and measurements of utility. Iatreia. 2011; 24(4):365-77.

14. Valderas JM, Ferrer M, Alonso J. Instrumentos de medida de calidad de vida relacionada con la salud y de otros resultados percibidos por los pacientes. Med Clínica. 2005;125, Supplement 1:56-60.

15. Velarde-Jurado E, Avila-Figueroa C. Evaluación de la calidad de vida. Salud Pública Méx. 2002; 44(4):349-61.

16. Vilagut G, Ferrer M, Rajmil L, Rebollo P, Permanyer G. El Cuestionario de Salud SF-36 español: una década de experiencia y nuevos desarrollos. Gac Sanit. 2005; 19(2):135-50.

17. Calvo F, Costa AM, García-Conde J, Megía MJ. Sin reconocimiento recíproco no hay calidad asistencial. Rev Esp Salud Pública. 2011; 85(5):459-68.

18. Tuesca R. La Calidad de Vida, su importancia y cómo medirla. Salud Uninorte. 2005; (21):76-86.

19. Vinaccia S, Quiceno JM. Calidad de vida relacionada con la salud y factores psicológicos: un estudio desde la enfermedad pulmonar obstructiva crónica - EPOC. Ter Psicológica. 2011; 29(1):65-75.

20. Vinaccia S, Quiceno JM, Fonseca P, Fernández H, Tobón S. Calidad de vida relacionada con la salud y su impacto sobre la cognición hacia la enfermedad en pacientes con hiperplasia prostática. Acta Colomb Psicol. 2006; 9(2):47-56.

21. Guevara-Cuellar CA, Takeuchi-Tan Y. Factors associated with quality of life in patients with carpal tunnel syndrome. Acta Neurol Colomb. 2014; 30(2):82-8.

22. Henao AS, Pirela CT, Alonso LM, Álvarez A, Londoño M. Quality of life in patients post ischaemic cerebrovascular event in two hospitals of Barranquilla (Colombia). Salud. 2011; 25(1):73-9.

23. Vinaccia S, Tobón S, Moreno E, Cadena J, Anaya JM. Evaluación de la calidad de vida en pacientes con diagnóstico de artritis reumatoide. Int J Psychol Psychol Ther. 2005; 5(1):45-59.

24. Vinaccia S, Fernández H, Quiceno JM, López M, Otálvaro C. Calidad de Vida Relacionada con la salud y apoyo social funcional en pacientes diagnosticados con VIH/Sida. Ter Psicológica. 2008; 26(1):125-32.

25. Contreras F, Esguerra G, Espinosa JC, Gutiérrez C, Fajardo L. Calidad de vida y adhesión al tratamiento en pacientes con insuficiencia renal crónica en tratamiento de hemodiálisis. Univ Psychol. 2006; 5(3):487-99.

26. Vinaccia $S$, Quiceno JM, Fernández $H$, Pérez BE, Sánchez MO, Londoño A. Calidad de vida relacionada con la salud y apoyo social percibido en pacientes con diagnóstico de tuberculosis pulmonar. Anales de Psicología. 2007; 23(2):245-52.

27. Contreras F, Esguerra GA, Espinosa JC, Gómez V. Estilos de afrontamiento y calidad de vida en pacientes con insuficiencia renal crónica (IRC) en tratamiento de hemodiálisis. Acta Colombiana de Psicología. 2007; 10(2):169-79.

28. Vinaccia S, Quiceno JM, Fernández H, Contreras F, Bedoya M, Tobón S, et al. Calidad de vida, personalidad resistente y apoyo social percibido en pacientes con diagnóstico de cáncer pulmonar. Psicol Salud. 2014; 15(2):207-20.

29. Trejos AM, Mosquera M, Tuesca R. Niñez afectada con VIH/SIDA: calidad de vida, funcionalidad familiar y apoyo social en cinco ciudades colombianas. Salud Uninorte. 2009; 25(1):17-32.

30. Vinaccia S, Margarita J, Gómez LA, Montoya M. Calidad de vida relacionada con la salud en pacientes con diagnóstico de hipertensión arterial leve. Diversitas: Perspectivas en Psicología. 2007; 3(2):203-11.

31. Ariza K, Isaza P, Gaviria AM, Quiceno JM, Vinaccia S, Alvarán L, et al. Calidad de vida relacionada con la salud, factores psicológicos y fisiopatológicos en pacientes con diagnóstico de Lupus Eritematoso Sistémico - LES. Terapia Psicológica. 2010; 28(1):27-36.

32. Vinaccia S, Fernández H, Escobar O, Calle E, Andrade IC, Contreras F, et al. Calidad de vida y conducta de enfermedad en pacientes con diabetes mellitus tipo II. Suma Psicológica. 2006; 13(1):15-31.

33. Rojas A, Zapata A, Anaya J, Pineda R. Predictores de calidad de vida en pacientes con fibromialgia. Asociación Colombiana de Reumatologia. 2005; 12(4):295-300.

34. Cardona-Arias JA, Franco-Aguirre JQ. Efecto de la psoriasis sobre calidad de vida relacionada con la salud: meta-análisis 2003-2013. Rev Argent Dermatol. 2014; 95(2):5-15.

35. Franco-Aguirre J, Cardona Arias J. Calidad de vida relacionada con la salud en personas con artritis reumatoide: caracterización de los estudios publicados entre 2003-2013. IATREIA. 2015; 28(2):109-19.

36. Delgado-Sanz MC, Prieto-Flores ME, Forjaz MJ, Ayala A, Rojo-Pérez F, Fernández-Mayoralas $\mathrm{G}$, et al. Influencia de los problemas crónicos de salud en las dimensiones del cuestionario EQ-5D: estudio en personas mayores institucionalizadas y no institucionalizadas. Rev Esp Salud Pública. 2011; 85(6):555-68. 
37. Instituto de Evaluación Tecnológica en Salud. Manual para la elaboración de evaluaciones económicas en salud. Bogotá D.C.: IETS; 2014.

38. Herdman M, Badia X, Berra S. El EuroQol-5D: una alternativa sencilla para la medición de la calidad de vida relacionada con la salud en atención primaria. Atención Primaria. 2001; 28(6):425-9.

39. Clasificación Internacional de Enfermedades. Organización Mundial de la Salud [Internet]. [citado 9 de octubre de 2018]. Disponible en: https://www.paho.org/col/index.php?option=com_con tent\&view=article\&id=535:clasificacion-internacional-de-enfermedades\&Itemid $=487$.

40. Organización Mundial de la Salud. Enfermedades crónicas [Internet]. [citado 9 de octubre de 2018]. Disponible en: http://www.who.int/topics/chronic_diseases/es/.

41. Institute of Medicine. Living well with chronic illness: A call for public health action. Washington, DC: The National Academies Press. 2012.

42. Organización Mundial de la Salud. Prevención de las enfermedades crónicas [Internet]. [citado 9 de octubre de 2018]. Disponible en: http://www.who.int/chp/chronic_disease_report/part1/ es/index1.html.

43. Institute for Health Metrics and Evaluation, Human Development Network, The World Bank. La carga mundial de morbilidad: generar evidencia, orientar políticas - Edición regional para América Latina y el Caribe. Seattle, WA: IHME, 2013.

44. Grupo de trabajo para la actualización del Manual de Elaboración de GPC. Elaboración de Guías de Práctica Clínica en el Sistema Nacional de Salud. Actualización del Manual Metodológico. 2016 [Internet]. Disponible en: http://portal.guiasalud.es/emanuales/ elaboracion_2/?capitulo=9.

45. Matesanz M, Íñiguez I, Rubal D, Pértega S, Conde J, Casariego E. Multimorbilidad en los servicios médicos hospitalarios: un problema clínico y de gestión. Galicia Clin. 2012; 73(Supl. 1):S15-S19.

46. Fortin M, Lapointe L, Hudon C, Vanasse A, Ntetu AL, Maltais D. Multimorbidity and quality of life in primary care: A systematic review. Health Qual Life Outcomes. 2004; 2:51.

47. Zapatero A, Barba R, Grupo Gestión FEMI. Estudio de un millón de altas hospitalarias en Medicina Interna [internet]. [citado 9 de octubre de 2018]. Disponible en: www.fesemi.org/ grupos/gestionclinica/noticias/un millon de altas.pdf.

48. Cerqueiro JM, Terrón F, Abeledo C, López MJ, Matesanz M, Casariego E. Resultados asistenciales de una consulta monográfica para pacientes con insuficiencia cardíaca. Estudio de cohortes. Galicia Clin. 2010; 71(3):103-10.

49. Kulczycka L, Sysa-Jedrzejowska A, Robak E. Quality of life and satisfaction with life in SLE patients - the importance of clinical manifestations. Clin Rheumatol. 2010; 29(9):991-7. 